\title{
Retrieval of the full magnetic vector with the He I multiplet at $1083 \mathrm{~nm}$
}

\section{Maps of an emerging flux region}

\author{
A. Lagg, J. Woch, N. Krupp, and S. K. Solanki ${ }^{1}$
}

Max-Planck-Institut für Aeronomie, Max-Planck-Str. 2, Katlenburg-Lindau, Germany

Received 9 July 2003 / Accepted 21 October 2003

\begin{abstract}
A technique is presented to invert Stokes profiles of the He I $1083 \mathrm{~nm}$ multiplet lines in order to obtain the full magnetic vector and the line-of-sight velocity. The technique makes use of spectropolarimetry connected with the Zeeman effect supplemented by a simple Hanle effect based diagnostic when appropriate. It takes into account effects like line saturation, magnetooptical effects, etc. and is coupled with a genetic algorithm, which ensures that the global minimum in a goodness of fit hypersurface is found. Tests using both artificial and real data demonstrated the robustness of the method. As an illustration maps of deduced parameters of an emerging flux region are shown and briefly discussed.
\end{abstract}

Key words. techniques: polarimetric - methods: data analysis - Sun: chromosphere - Sun: infrared - Sun: magnetic field

\section{Introduction}

Whereas the magnetic field of the Sun manifests itself most dominantly in the solar corona, the majority of observations of the field have in the past been restricted to the solar photosphere. This has mainly to do with the relative simplicity of magnetographic and spectropolarimetric observations in visible and infrared spectral lines formed in the photosphere (Stenflo et al. 1984; Jones et al. 1992; Solanki et al. 1992; Lites et al. 1993; Scherrer et al. 1995; Collados 2001).

The magnetic field in the upper solar atmosphere can be determined, for example, through extrapolations from the photosphere (Seehafer \& Staude 1979; Falconer et al. 1997; Yan \& Sakurai 1997; Schrijver et al. 1999; Régnier et al. 2002) or from cyclotron radiation recorded at radio wavelengths in sufficiently strong fields (White \& Kundu 1997). The first technique is, despite many advances, still uncertain in several decisive details and requires improved measurements in the upper atmosphere to test and calibrate it. The second technique only provides the field strength and maps require considerable time to build up, so that temporal and spatial detail is lost. It has nonetheless been a major empirical source of magnetic field measurements in the solar corona (White 2002). Other examples of diagnostic tools that have been developed for investigating the magnetic field vector in the outer regions of the solar atmosphere are the non-LTE inversion of Stokes profiles induced by the Zeeman effect (Socas-Navarro et al. 2000b), which has been applied to explore the dynamical and

Send offprint requests to: A. Lagg,

e-mail: lagg@linmpi.mpg.de magnetic properties of sunspot chromospheres (Socas-Navarro et al. 2000a), the Hanle and Zeeman effects in the $\mathrm{D}_{3}$ line of He I (Landi Degl'Innocenti 1982), including a PCA-based algorithm for facilitating the inversion of $\mathrm{D}_{3}$ Stokes profiles observed in prominences (López Ariste \& Casini 2002), and the Hanle and Zeeman effect in the He I $1083 \mathrm{~nm}$ multiplet with applications to both solar coronal filaments and prominences (Trujillo Bueno et al. 2002).

It is particularly important to map the full magnetic vector in the corona at a good spatial resolution, since above the photosphere the magnetic field is expected to become increasingly inhomogeneous in direction and has been proposed to form tangential discontinuities (Parker 1983a,b, 1988). If this is indeed the case, then magnetic reconnection at such sites would be a major source of coronal heating. So far, however, the magnetic structure of loops and the presence of tangential discontinuities have been deduced indirectly, from intensity images and extrapolations of the photospheric field.

In the present paper we consider spectropolarimetry of the He I $1083 \mathrm{~nm}$ multiplet which is susceptible to both the Zeeman and Hanle effects, as shown clearly by Trujillo Bueno et al. (2002). Among the several physical effects demonstrated in the just quoted letter, the one we want to emphasize here is that the Hanle effect in forward scattering at the solar disc center creates linear polarization in the He I $1083 \mathrm{~nm}$ multiplet, which can also be understood within the framework of the oscillator model for the Hanle effect (Trujillo Bueno 2001). In the absence of magnetic fields (or in the presence of a vertical magnetic field) forward scattering processes at the solar disc 
center do not produce linear polarization in the $1083 \mathrm{~nm}$ multiplet. However, in the presence of an inclined magnetic field that breaks the symmetry of the scattering polarization problem at the solar disc center, forward scattering processes can produce measurable linear polarization in lines like those of the He I $1083 \mathrm{~nm}$ multiplet. The largest linear polarization amplitudes are expected for horizontal magnetic fields having strengths above a few Gauss only (Trujillo Bueno 2003). Obviously, if the magnetic strength is "sufficiently large" (e.g., larger than $200 \mathrm{G}$ ) the linear polarization signal would be dominated by the transverse Zeeman effect. On the other hand, the circular polarization Stokes $V$ signals of the He I $1083 \mathrm{~nm}$ multiplet are practically always dominated by the longitudinal Zeeman effect.

Magnetography using the He I $1083 \mathrm{~nm}$ multiplet was pioneered by Harvey \& Hall (1971), who showed that the magnetic field near the base of the corona, as sampled by these lines (e.g. Avrett et al. 1994), is more homogeneous than the photosphere. Thereafter these lines lay dormant as magnetic diagnostics until Rüedi et al. (1995) and Penn \& Kuhn (1995) carried out the first spectropolarimetric observations of Stokes $I$ and $V$ and showed the major advantages of analyzing these lines due to their optical thinness, their narrowness and the absence of any significant photospheric contribution. However, due to the expected large range of inclinations and azimuths of the magnetic field in the corona it is particularly necessary to measure the full magnetic vector in the upper solar atmosphere. Rüedi et al. (1996) and Lin et al. (1998) demonstrated that such measurements are feasible. However, the lack of appropriate instrumentation hindered the earlier use of this diagnostic. The Tenerife Infrared Polarimeter (TIP) (Martínez Pillet et al. 1999) at the German Vacuum Tower Telescope (VTT) at the Spanish observatory of Izaña (Tenerife) provides the necessary data to realize the potential of this diagnostics. In fact, this is the infrared polarimeter used by Trujillo Bueno et al. (2002) to demonstrate the diagnostic interest of the Hanle effect in the He I $1083 \mathrm{~nm}$ multiplet at the solar disc center via spectropolarimetric observations of solar coronal filaments. These authors applied the quantum theory of line formation, as developed by Landi Degl'Innocenti (1982), taking into account both the Hanle and Zeeman effects with optical pumping processes in multilevel atomic models, and inferred the full magnetic field vector of solar prominences and filaments via spectropolarimetry of the He I $1083 \mathrm{~nm}$ multiplet. In order to be able to model the Stokes profiles observed in filaments, where the helium lines are seen in absorption, Trujillo Bueno et al. (2002) took into account radiative transfer effects in slabs anisotropically illuminated from "below" by the photospheric radiation field.

There is clearly a need to further improve the analysis procedures for the He I multiplet before their undoubted potential can be realized. This is precisely the aim of the present paper, which introduces a simple Milne-Eddington model for describing the radiative transfer in these lines (Sect. 3). Since the lines are almost optically thin, this approach should be adequate for determining magnetic and velocity parameters. Since we are mainly interested in active regions, we account for both the transverse and longitudinal Zeeman effects as explained
Table 1. Line parameters (atomic parameters taken from the NIST Atomic Spectra Database.

\begin{tabular}{lcccc}
\hline \hline Line & $\begin{array}{c}\text { wavelength } \\
{[\mathrm{nm}]}\end{array}$ & transition & $\begin{array}{c}\text { effective } \\
\text { Landé } \\
\text { factor } g_{\text {eff }}\end{array}$ & $\begin{array}{c}\text { relative } \\
\text { oscillator } \\
\text { strength }\end{array}$ \\
\hline He Ia & 1082.90911 & $2 s^{3} S_{1}-2 p^{3} P_{0}$ & 2.0 & 0.09 \\
$\mathrm{He} \mathrm{Ib}$ & 1083.02501 & $2 s^{3} S_{1}-2 p^{3} P_{1}$ & 1.75 & 0.30 \\
$\mathrm{He} \mathrm{Ic}$ & 1083.03397 & $2 s^{3} S_{1}-2 p^{3} P_{2}$ & 0.875 & 0.60 \\
$\mathrm{Si} \mathrm{I}$ & 1082.70880 & $4 s^{3} P_{2}^{0}-4 p^{3} P_{2}$ & 1.5 & \\
$\mathrm{Ca} \mathrm{I}$ & 1082.93 & $4 p^{3} F_{3}^{0}-6 d^{3} D_{2}$ & 1.0 & \\
\hline
\end{tabular}

in Sect. 3.1 (i.e., by accounting for the Zeeman splitting but neglecting optical pumping processes). However, since in our observations of emerging flux regions we have detected some linear polarization signals that are clearly caused by the same Hanle effect in forward scattering discussed by Trujillo Bueno et al. (2002), we have included in our inversion code a simple strategy for dealing with it in observations taken not far away from the solar disc center. In Sect. 4 we present a new approach to inversions of solar spectropolarimetric data involving genetic algorithms. These techniques are tested in Sect. 5 and applied to data obtained in an emerging flux region (data described in Sect. 2, results of the inversion in Sect. 6; further results are given by Solanki et al. 2003). Finally, an outlook is presented in Sect. 7.

\section{Instrument and observations}

An active region in the course of emergence (NOAA 9451), located at $33^{\circ} \mathrm{W}, 22^{\circ} \mathrm{S}$, i.e. at $\mu=\cos \Theta=0.8$ (where $\Theta$ is the heliocentric angle, the angle between the line-of-sight and the solar surface normal), was recorded with the Tenerife Infrared Polarimeter mounted behind the Echelle spectrograph on the Vacuum Tower Telescope at the Teide observatory on Tenerife. The Echelle spectrograph allows for a spectral resolution of $30 \mathrm{~m} \AA$ per pixel, while the pixel size is $0.4^{\prime \prime}$. The active region was covered by scanning in steps of $0.4^{\prime \prime}$ perpendicular to the slit orientation. The number of spatial pixels along the slit and the scan steps determine the size of the observed region $\left(46 \times 30 \mathrm{Mm}^{2}\right)$. Atmospheric turbulence limited the spatial resolution of the data set considered for this analysis to $1.5^{\prime \prime}$. The exposure time of $\approx 5 \mathrm{~s}$ per slit position led to a noise level of typically $\approx 5 \times 10^{-4}$. The observed wavelength range from 1082.5 to $1083.3 \mathrm{~nm}$ contains two photospheric lines of Si I and Ca I, the chromospheric He I multiplet and a telluric blend at $1083.2 \mathrm{~nm}$ (see Table 1).

Figure 1 displays observed maps of the four Stokes parameters. The maps were obtained by integrating the absolute values of the Stokes vectors within a wavelength range of $\pm 100 \mathrm{~m} \AA$ around the He Ic line. The $I$ and the $V$ profiles clearly indicate the presence of two pores with high Stokes $V$ signal. These pores are also visible in the slit-jaw image and in a continuum map of the region (Solanki et al. 2003). The presence of dark striations parallel to the line connecting the 


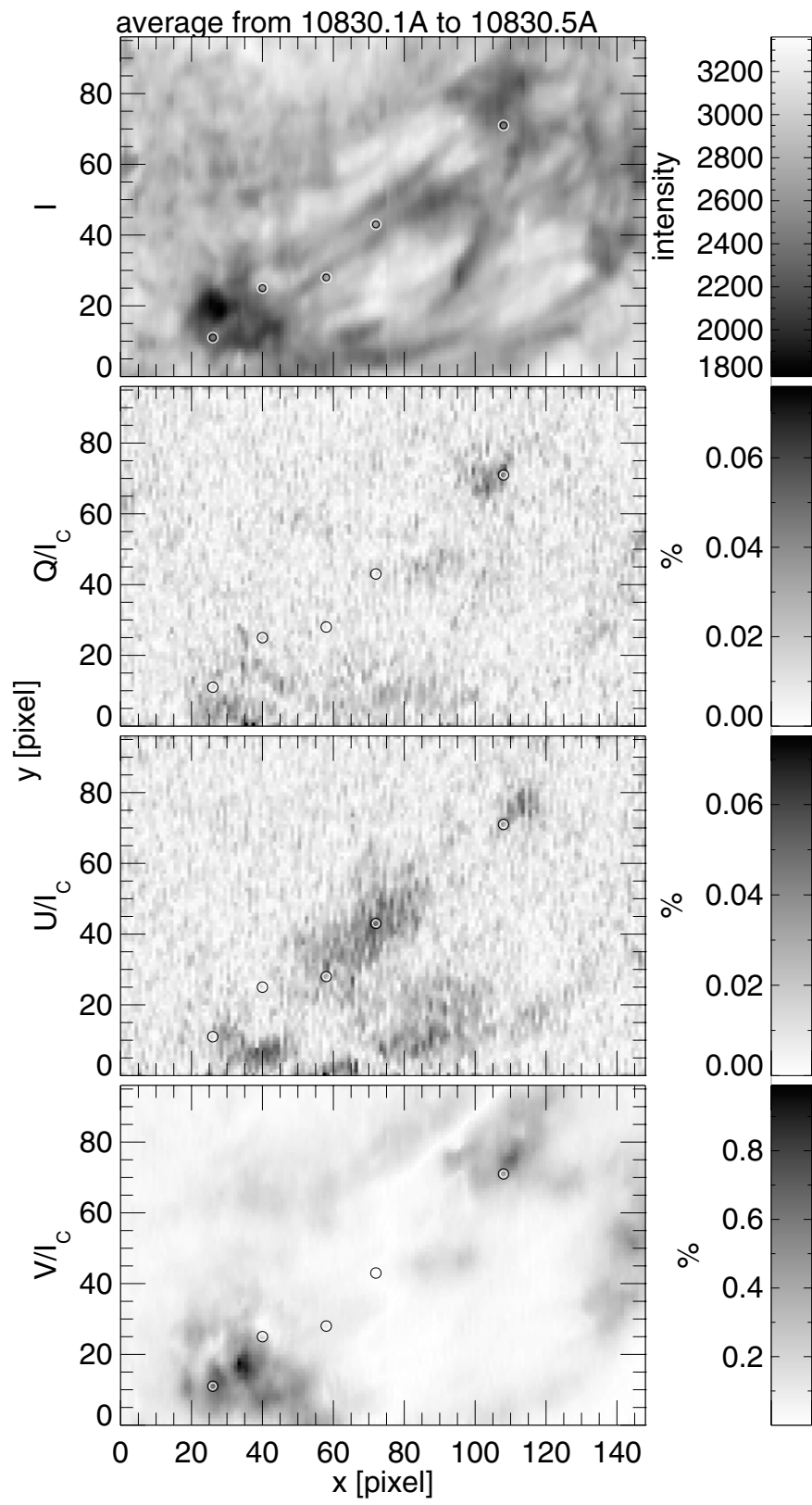

Fig. 1. Stokes $I, Q, U$ and $V$ maps of the observed region (NOAA 9451 , solar position $33^{\circ} \mathrm{W}, 22^{\circ} \mathrm{S}$ ). Plotted are the absolute values of the wavelength integrated Stokes parameters of the strongest He I lines normalized to the continuum intensity. The size of the region is $46 \times 30 \mathrm{Mm}$. The white / black circles indicate the positions where a detailed analysis of spectra is discussed in this paper. The reference direction for Stokes $Q$ is along the $+y$-axis of the maps.

two pores (seen in white light) and the fibril-like structures in the He I Stokes I map indicate that sub-surface magnetic flux tubes were emerging into the solar atmosphere at the time of the observations. Here they form magnetic loops connecting the two pores of opposite magnetic polarity. The Stokes $U$ map shows two patches of a relatively strong signal above the site of emergence. We expect that this signal is coming from near the top of the loops connecting the opposite polarity footpoints. This signal is caused by scattering-polarization influenced by

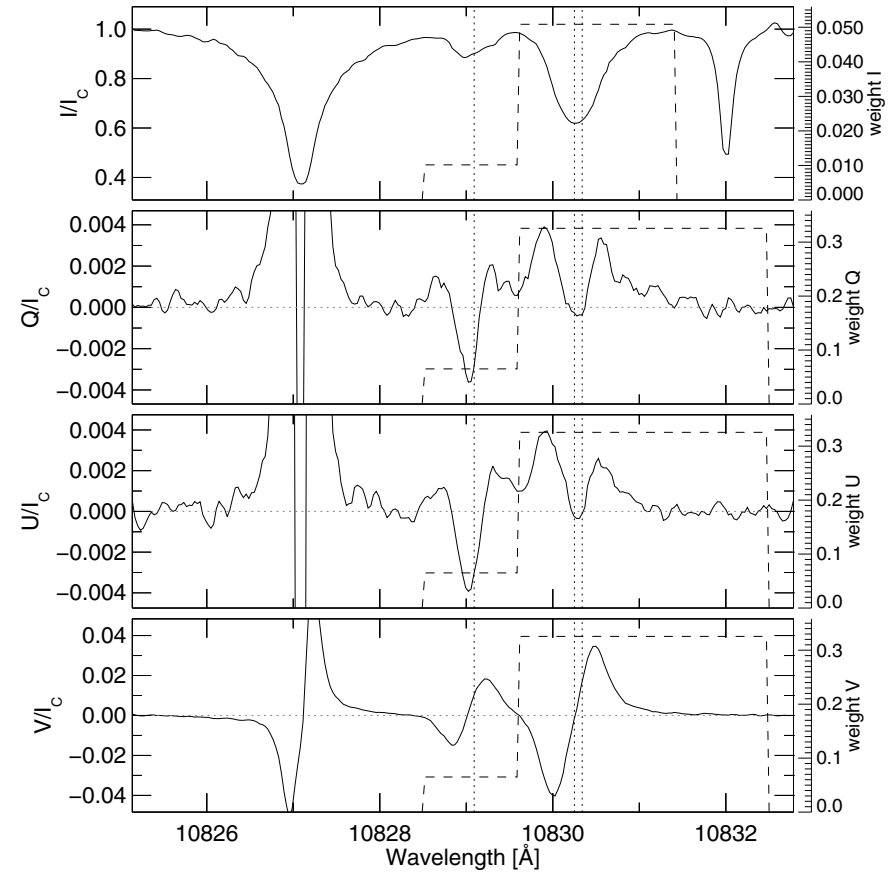

Fig. 2. Average Stokes $I, Q, U$ and $V$ spectrum at position $x=108$, $y=71$ ). For later reference the weighting scheme is indicated by the dashed lines (see Sect. 4). The reference direction for Stokes $Q$ is along the $+y$-axis of the maps in Fig. 1 .

the Hanle effect and dominates over the signal from the Zeeman effect at these locations.

Figure 2 presents a spectrum averaged over 6 pixels around position $x=108, y=71$ in Fig. 1. All four Stokes parameters of the He I multiplet show a clear Zeeman-signal well above the noise-level of $\approx 5 \times 10^{-4}$. The dotted vertical lines indicate the positions of the central wavelengths of the He I lines. Although the Stokes $V$ signals due to the Si I line and the He I multiplet have the same amplitudes within a factor of 2 of each other, the linear polarization signal of the Si I line is about an order of magnitude higher than of the $\mathrm{He} \mathrm{Ib}$ and $\mathrm{He}$ Ic blend $\left(Q / I_{C} \approx U / I_{C} \approx 0.05\right)$. Note that the He Ia line is blended by a photospheric $\mathrm{Ca}$ I line at almost the same central wavelength (see Table 1). The analysis therefore concentrates on the $\mathrm{He} \mathrm{Ib}$ and He Ic lines which are free of blends. For highly redshifted He profiles the telluric blend at $1083.2 \mathrm{~nm}$ must be excluded from the analysis.

\section{Description of the Stokes profiles}

\subsection{Zeeman-split profiles}

The analysis of the photospheric Si I line and the chromospheric He I lines is based on an inversion involving the forward calculation of the four Stokes profiles. The Si I line was analyzed using the sophisticated and robust inversion code SPINOR (Frutiger 2000; Frutiger et al. 2000). This code solves the Unno-Rachkowsky radiative transfer equations in LTE (local thermodynamic equilibrium) conditions and retrieves for an arbitrary number of atmospheric components parameters like temperature, magnetic field strength and direction, 
line-of-sight-velocity, as a function of optical depth, as well as depth independent micro- and macro-turbulence.

For the He I multiplet this code is not applicable since these lines are formed under non-LTE conditions in the upper chromosphere at a height of $\gtrsim 1500 \mathrm{~km}$ above the photosphere. Given that these lines have a very complex formation, even in a simple plane-parallel atmosphere (Avrett et al. 1994) and that the structure of the upper chromosphere may be rather complicated, at a convoluted coronal boundary (evidence for this is presented by Solanki et al. 2003), we refrain from carrying out a time consuming non-LTE analysis. As pointed out by Rüedi et al. (1995) the fact that the lines are (almost) optically thin and obtain most of their contribution from the upper chromosphere means that they can be used to deduce the magnetic field and line-of-sight velocity at the height of formation of the lines without the need to understand the details of their formation.

Our previous work concentrated on the straightforward method of fitting sums of Gaussians to observed Stokes $I$ and $V$ profiles. In a first step we extended this procedure to fitting all 4 Stokes profiles. These fits showed promise for a stable determination of magnetic field strength and direction, in particular when combined with the appropriate formulae for the azimuth, $\chi$, and the inclination to the line-of-sight, $\gamma$ (Auer et al. 1977):

$$
\begin{aligned}
& \tan 2 \chi=\frac{U}{Q}, \\
& \frac{\sin ^{2} \gamma}{2 \cos \gamma} \approx \frac{Q \cos 2 \chi+U \sin 2 \chi}{V} .
\end{aligned}
$$

However, this approach neglects magnetooptical effects, which are strongest for $Q$ and $U$, and line saturation, which, although small in the He I multiplet (Giovanelli \& Hall 1977), nevertheless most strongly affects Stokes $Q$ and $U$ through the ratio of the $\sigma$ - to the $\pi$-component amplitude (e.g. Landi Degl'Innocenti 1976; Solanki et al. 1987). Finally, Eq. (2) is strictly valid only for completely Zeeman-split lines, so that it is expected to introduce systematic errors into the deduced $\gamma$, in particular when the field is nearly transverse.

Therefore, we have taken a somewhat more sophisticated approach that includes magnetooptical effects, the basics of radiative transfer (e.g. Jefferies et al. 1989; del Toro Iniesta 2003), as well as allowing for the Hanle effect in a simple manner, which, however, is adequate for the current data set.

The spectral shape of a single Zeeman component of a spectral line can be expressed as a convolution of the Lorentzian absorption and the dispersion profiles with a Gaussian, resulting in a Voigt function $H$ and FaradayVoigt function $F$, respectively. Following the notation by Landi Degl'Innocenti (1976) the resulting absorption profiles $\eta$ and the anomalous dispersion profiles $\rho$ are given by

$$
\begin{array}{cl}
\eta_{b, r}=\eta_{0} H\left(a, v \pm v_{B}\right), & \rho_{b, r}=2 \eta_{0} F\left(a, v \pm v_{B}\right), \\
\eta_{p}=\eta_{0} H(a, v), & \rho_{p}=2 \eta_{0} F(a, v),
\end{array}
$$

where $\eta_{0}$ is the ratio of the line center to the continuum opacity, $a$ is the damping constant of the spectral line, $v$ is the wavelength separation to the central wavelength, $\lambda_{0}$, relative to the
Doppler shift, $\Delta \lambda_{v_{\mathrm{LOS}}}$, and $v_{B}$ is the Zeeman splitting in units of the Doppler width $\Delta \lambda_{\mathrm{D}}$ :

$v=\frac{\lambda-\lambda_{0}-\Delta \lambda_{v_{\mathrm{LOS}}}}{\Delta \lambda_{\mathrm{D}}}, v_{B}=\frac{\Delta \lambda_{B}}{\Delta \lambda_{\mathrm{D}}}, a=\Gamma \frac{\lambda_{0}^{2}}{4 \pi \Delta \lambda_{\mathrm{D}}}$,

where $\Gamma$ is the damping factor in units of the velocity of light. The indices $b$ and $r$ denote the blue and the red $\sigma$-components of the Zeeman triplet, the central $\pi$ component being indicated by the index $p$. The Zeeman-splitting $\Delta \lambda_{B}$ (in $\AA$ ) due to the magnetic field strength is given by

$\Delta \lambda_{B}=4.67 \times 10^{-13} g_{\mathrm{eff}} B \lambda^{2}$,

where $g_{\text {eff }}$ is the effective Landé factor and $B$ is the magnetic field strength in Gauss. Test runs showed that the use of an effective Landé factor instead of the calculation of all Zeeman components separately returned very similar results at a gain of almost a factor of three in computation time.

The profiles $\eta_{b, p, r}$ and $\rho_{b, p, r}$ are combined to form the absorption profiles

$$
\begin{aligned}
\eta_{I} & =\frac{1}{2}\left[\left(\frac{\eta_{r}+\eta_{b}}{2}\right)\left(1+\cos ^{2} \gamma\right)+\eta_{p} \sin ^{2} \gamma\right], \\
\eta_{Q} & =\frac{1}{2}\left(\eta_{p}-\frac{\eta_{r}+\eta_{b}}{2}\right) \sin ^{2} \gamma \cos 2 \chi \\
\eta_{U} & =\frac{1}{2}\left(\eta_{p}-\frac{\eta_{r}+\eta_{b}}{2}\right) \sin ^{2} \gamma \sin 2 \chi \\
\eta_{V} & =\left(\frac{\eta_{r}-\eta_{b}}{2}\right) \cos \gamma
\end{aligned}
$$

and the dispersion profiles

$\rho_{Q}=\frac{1}{2}\left(\rho_{p}-\frac{\rho_{r}+\rho_{b}}{2}\right) \sin ^{2} \gamma \cos 2 \chi$,

$\rho_{U}=\frac{1}{2}\left(\rho_{p}-\frac{\rho_{r}+\rho_{b}}{2}\right) \sin ^{2} \gamma \sin 2 \chi$,

$\rho_{V}=\left(\frac{\rho_{r}-\rho_{b}}{2}\right) \cos \gamma$

of the individual Stokes parameters. These profiles are the elements of the propagation matrix

$\mathbf{K}=\left(\begin{array}{cccc}\eta_{I} & \eta_{Q} & \eta_{U} & \eta_{V} \\ \eta_{Q} & \eta_{I} & \rho_{V} & -\rho_{U} \\ \eta_{U} & -\rho_{V} & \eta_{I} & \rho_{Q} \\ \eta_{V} & \rho_{U} & -\rho_{Q} & \eta_{I}\end{array}\right)$.

The angles $\chi$ and $\gamma$ are the azimuthal and the inclination angle of the magnetic field vector with respect to the line-of-sight. In the case of a Zeeman triplet the function $\eta_{p}$ ( $\pi$-component of the Zeeman triplet) is evaluated at the wavelength $\lambda=$ $\lambda_{0}-\Delta \lambda_{v_{\text {LOS }}}$, the $\sigma$-components $\eta_{b}$ (left circular polarization) and $\eta_{r}$ (right circular polarization) are evaluated at $\lambda=\lambda_{0}-$ $\Delta \lambda_{v_{\text {LOS }}} \pm \Delta \lambda_{B}$, where $\Delta \lambda_{v_{\text {LOS }}}$ is the Doppler shift due to the motion of the atom along the line-of-sight. For more complex splitting patterns the shifts and strengths of the Zeeman components can be computed following e.g. Condon \& Shortley (1963) or Landi Degl'Innocenti (1976).

To calculate the Stokes profiles we assume the simplest case for the solution of the radiative transfer equation, which is a height-independent propagation matrix (Milne-Eddington 
atmosphere). This is adequate for the weak saturation shown by the He I lines and their relatively narrow height of formation in standard atmospheres (Avrett et al. 1994). Also it is reasonable given the lack of knowledge of the true conditions in the formation region of these lines and has the advantage of being efficient to compute. All quantities of the atmosphere are assumed to be constant with optical depth except for the source function, which varies linearly with optical depth: $S(\tau)=S_{0}+S_{1} \tau$. Note that in this case the source function can differ strongly from the Planck function and needs not be known in advance since $S_{0}$ and $S_{1}$ can be obtained from the line profiles. This leads to explicit expressions for the 4 Stokes parameters (all normalized to $I_{\mathrm{c}}$ ), also known as the Unno-Rachkowsky solution of the transfer equation (Unno 1956; Rachkowsky 1962, 1967):

$$
\begin{aligned}
I= & S_{0}+\frac{\mu S_{1}}{\Delta}\left(1+\eta_{I}\right)\left[\left(1+\eta_{I}\right)^{2}+\rho_{Q}^{2}+\rho_{U}^{2}+\rho_{V}^{2}\right] \\
Q= & -\frac{\mu S_{1}}{\Delta}\left[\left(1+\eta_{I}\right)^{2} \eta_{Q}\right. \\
& \left.+\left(1+\eta_{I}\right)\left(\eta_{V} \rho_{U}-\eta_{U} \rho_{V}\right)+\rho_{Q} R\right] \\
U= & -\frac{\mu S_{1}}{\Delta}\left[\left(1+\eta_{I}\right)^{2} \eta_{U}\right. \\
& \left.+\left(1+\eta_{I}\right)\left(\eta_{Q} \rho_{V}-\eta_{V} \rho_{Q}\right)+\rho_{U} R\right] \\
V= & -\frac{\mu S_{1}}{\Delta}\left[\left(1+\eta_{I}\right)^{2} \eta_{V}\right. \\
& \left.+\left(1+\eta_{I}\right)\left(\eta_{U} \rho_{Q}-\eta_{Q} \rho_{U}\right)+\rho_{V} R\right]
\end{aligned}
$$

The abbreviations $\Delta$ and $R$ are defined as follows:

$$
\begin{aligned}
\Delta= & \left(1+\eta_{I}\right)^{2}\left[\left(1+\eta_{I}\right)^{2}-\eta_{Q}^{2}-\eta_{U}^{2}-\eta_{V}^{2}\right. \\
& \left.+\rho_{Q}^{2}+\rho_{U}^{2}+\rho_{V}^{2}\right]-R^{2} \\
R= & \eta_{Q} \rho_{Q}+\eta_{U} \rho_{U}+\eta_{V} \rho_{V} .
\end{aligned}
$$

The source function at $\tau=0$ (solar surface) was eliminated as a free parameter because the intensity of the unpolarized signal $I$ outside the line must equal the observed continuum intensity $I_{\mathrm{c}}$ :

$S_{0}+\mu S_{1}=I_{\mathrm{c}}$.

This forward calculation of the Stokes profiles leads to the following free parameters: magnetic field strength $B$ and direction (inclination angle $\gamma$, azimuthal angle $\chi$ ), line-of-sight velocity $v_{\mathrm{LOS}}$, Doppler width $\Delta \lambda_{\mathrm{D}}$, damping constant $a$, the ratio of the line center to the continuum intensity $\eta_{0}$ and the slope of the source function $S_{1}$. We define a set of these 8 parameters as an atmospheric component. We use the term component since we can allow for multiple such components within a resolution element to describe fine-structures unresolved by the observations. Due to the limited spatial resolution of the observations multiple components may well be present in some resolution elements which leads to an increase of the number of free parameters. These atmospheric components are mixed via a filling factor, $\alpha$, defining the contribution of an atmospheric component to the total observed profile. The sum of the filling factors of all atmospheric components within a resolution element is required to be unity. To limit the number of free parameters in a multi-component atmosphere we allow for coupling between different parameters. An example of a two-component

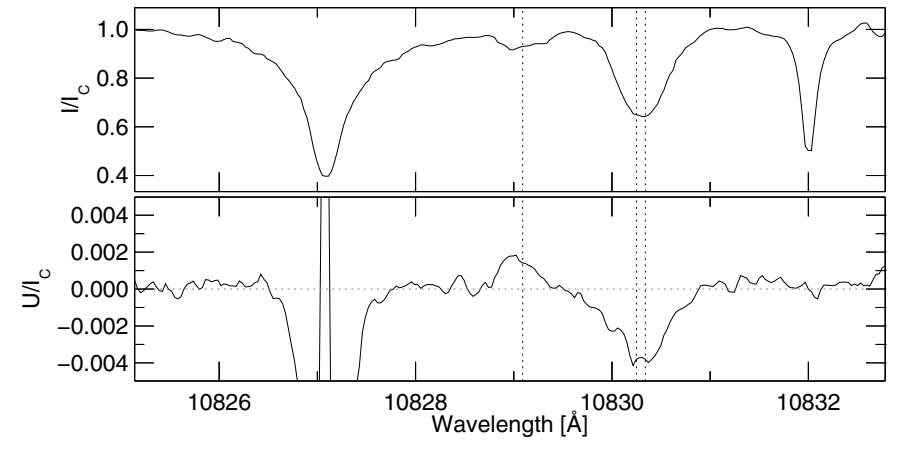

Fig. 3. Typical profile in the region dominated by scatteringpolarization (pixel $x=72, y=43$ ). The shape of the absorption signal of the He I lines in Stokes $U$ is similar to their $I$ profiles justifying the simplifying assumption made for the implementation of the Hanle effect. The horizontal dashed line represents $U=0$. The reference direction for Stokes $Q$ is along the $+y$-axis of the maps in Fig. 8 .

atmosphere consisting of a slow and a fast downflow component is discussed in Sect. 6.2.

Another assumption aimed at limiting the proliferation of free parameters is that all three He I lines are formed under identical conditions in the solar atmosphere. This assumption is well justified since the lines originate from the same multiplet. Thus, all free parameters except the line center absorption coefficient, $\eta_{0}$, and the source function gradient, $S_{1}$, are taken to be equal for the three He I lines. $\eta_{0}$ is scaled with the relative oscillator strength $f$ (see Table 1) for each He I line, so that it also needs to be determined only once for the whole multiplet. To take into account the blending of the He I lines the $\eta_{I, Q, U, V}$ and $\rho_{Q, U, V}$ values of the three lines are added together prior to entering them into Eqs. (15)-(22).

\subsection{Hanle effect}

The maps in Fig. 1 clearly show two patches of strong signal in Stokes $U$ close to the polarity inversion line. The $U$-profiles in this region are characteristic for a polarization signature caused by resonant scattering processes and the Hanle effect (see Fig. 3). Degenerate atomic levels are populated anisotropically due to anisotropic radiation pumping (see e.g. Happer 1972; Stenflo 1994). This leads to atomic level polarization (atomic polarization) implying sinks and/or sources for linear polarization (Trujillo Bueno 2001). This polarization signal is modified by the Hanle effect, creating a complicated dependence of the linear polarization on magnetic field strength, direction and viewing geometry (Trujillo Bueno et al. 2002). The main observational characteristics, $Q$ or $U$ profiles of the same shape as Stokes $I$, but with opposite signs for the He Ia line and the $\mathrm{He} \mathrm{Ib} \& \mathrm{He}$ Ic blend, are clearly exhibited by the $U$ profile shown in Fig. 3. This rules out instrumental cross-talk from $I$ to $U$ as the source of this signal. This interpretation is supported by the fact that the $U$ profile of Si I, which is not expected to exhibit the Hanle effect, but should suffer equally from instrumental cross-talk, is well reproduced by profiles calculated assuming pure Zeeman effect and no cross-talk. The resulting $Q$ and $U$ signals, together with the observed Stokes 
$V$ profiles, can be used to infer the full magnetic field vector as done by Trujillo Bueno et al. (2002) for the case of solar prominences and filaments. For special geometries polarization diagrams can be used to determine the magnetic field orientation and strength (e.g. Bommier et al. 1991; Faurobert-Scholl 1992; Nagendra et al. 1998).

As mentioned in the introduction, in the special case of a line-of-sight direction perpendicular to the solar surface (disc center) and a magnetic field inclined with respect to the local vertical, the Hanle effect creates measurable linear polarization in the He I multiplet (Trujillo Bueno et al. 2002). As pointed out by Trujillo Bueno (2003), if we choose as reference direction for Stokes $Q$ the projection direction of the magnetic field vector on the solar surface and the magnetic field is more inclined than Van Vleck's angle $\left(54.73^{\circ}\right)$, then $Q<0$ in the He Ia line ("blue line") and $Q>0$ in the $\mathrm{He} \mathrm{Ib}$ and He Ic line (red lines). However, if instead of being nearly horizontal the field turns out to be less inclined than Van Vleck's angle then $Q<0$ in the "red lines" and $Q>0$ in the "blue line". Moreover, in order to have what we have just summarized, the He I $1083 \mathrm{~nm}$ multiplet must be in the saturation regime of the Hanle effect, which occurs for magnetic strengths larger than only a few Gauss. Under such circumstances, we may infer the azimuth of the magnetic field vector from

$\tan (2 \chi)=(U / Q)$

This relation may give reasonable results for observations taken not far away from the solar disc center (Trujillo Bueno 2003), and has been applied by Collados et al. (2003) to infer the magnetic field azimuth in several coronal filaments observed with TIP. The point is that in the absence of magnetic fields the amplitude of the scattering polarization signals rapidly go to zero when approaching the disc center. Thus, detection of a scattering polarization signal in the He I $1083 \mathrm{~nm}$ multiplet in observations close to the center of the solar disc is really indicative of the presence of an inclined magnetic field (Trujillo Bueno et al. 2002). Since our observations were carried out at $\mu=0.8$ (i.e., not far away from disc center) and since Stokes $V$ changes sign within the locations at which the Stokes $U$ profiles show the strong scattering signal, we believe that the basic conditions for employing this simple but useful strategy are fulfilled.

As we show in Sect. 4 the results obtained using Eq. (24) are consistent with those from the Zeeman mechanism. To correct for the scattering polarization we take the following approach:

- A Voigt-profile is fit to the Stokes I profile. This returns damping, Doppler broadening and line shift for the profiles used to correct Stokes $Q$ and $U$.

- The scattering-polarization signal is assumed to be of similar shape as the absorption profile in Stokes $I$ (see Fig. 3). Therefore a profile $h$ with a free amplitude $H_{0}$ but fixed values for damping, Doppler broadening and line shift (obtained from Stokes $I$ ) is fit to the $Q$ and $U$ signals. This fit is carried out simultaneously with the fitting of all other free parameters of the inversion.
- The amplitudes of the profiles for the three different He I lines scale according to their relative oscillator strengths $f$. The sign of the amplitudes for the "blue" and the "red" lines follows the rule described above (Van Vleck's angle).

- The amplitudes for different atmospheric components are coupled via their filling factors.

An example of a profile showing a Zeeman characteristic linear polarization signal combined with a typical signal for scattering-polarization is presented in Sect. 4.

\section{Implementation}

The fit to the observed profiles is based on a minimization of goodness of fit function $\delta$. The synthesized profiles are calculated according to Sect. 3 assuming an arbitrary initial atmosphere. The parameters of this atmosphere are then adjusted such that the synthesized profiles match the observations. To ensure that this optimization process finds the global minimum of $\delta$ in parameter space we use the genetic algorithm PIKAIA (Charbonneau 1995). We compared this method with standard minimization routines and found a superior robustness, at some cost to computation time, however. Since computation time is not so critical when the synthesized profiles are calculated using Milne-Eddington atmospheres we have given preference to the robustness of the fit. Note that genetic algorithms find the global minimum in the $\delta$ hypersurface in contrast to, e.g., maximum likelihood techniques such as the Levenberg-Marquardt or the UOBYQA (unconstrained optimization by quadratic approximation, Powell 2002) algorithms.

The function to minimize is defined as follows:

$$
\begin{aligned}
\delta= & \sum \omega_{I}\left(I_{\mathrm{obs}}-I_{\mathrm{syn}}\right) /\left(I_{\mathrm{c}} \sigma_{I}\right) \\
& +\sum \omega_{Q}\left(Q_{\mathrm{obs}}-Q_{\mathrm{syn}}\right) / \sigma_{Q} \\
& +\sum \omega_{U}\left(U_{\mathrm{obs}}-U_{\mathrm{syn}}\right) / \sigma_{U} \\
& +\sum \omega_{V}\left(V_{\mathrm{obs}}-V_{\mathrm{syn}}\right) / \sigma_{V}
\end{aligned}
$$

where the sum runs over $n$ wavelength points of the profiles, $I_{\mathrm{c}}$ is the continuum level, the subscripts "obs" and "syn" refer to observed and synthesized Stokes profiles respectively, $\sigma_{I, Q, U, V}$ are parameters defining the strength of the signal

$$
\begin{aligned}
& \sigma_{I}=\frac{1}{n} \sum_{\lambda}\left|I_{\mathrm{obs}} / I_{\mathrm{c}}-1\right|, \\
& \sigma_{Q, U, V}=\frac{1}{n} \sum_{\lambda}\left|(Q, U, V)_{\mathrm{obs}}\right|,
\end{aligned}
$$

and $\omega_{I, Q, U, V}$ are the wavelength dependent weighting functions. The spectral shape of the weighting function was chosen to reflect the peculiarities of the spectral region (see Fig. 2, dashed lines). The highest weight was given to the two He I lines $\mathrm{He} \mathrm{Ib}$ and He Ic, since they are not blended by any other solar or telluric line. For wavelengths less than $1082.96 \mathrm{~nm}$ we reduced the weight by a factor of 5 . This region contains the He Ia line which is blended by a Ca I line. All wavelength points below $1082.85 \mathrm{~nm}$ and above $1083.25 \mathrm{~nm}$ were not used for the fits. 


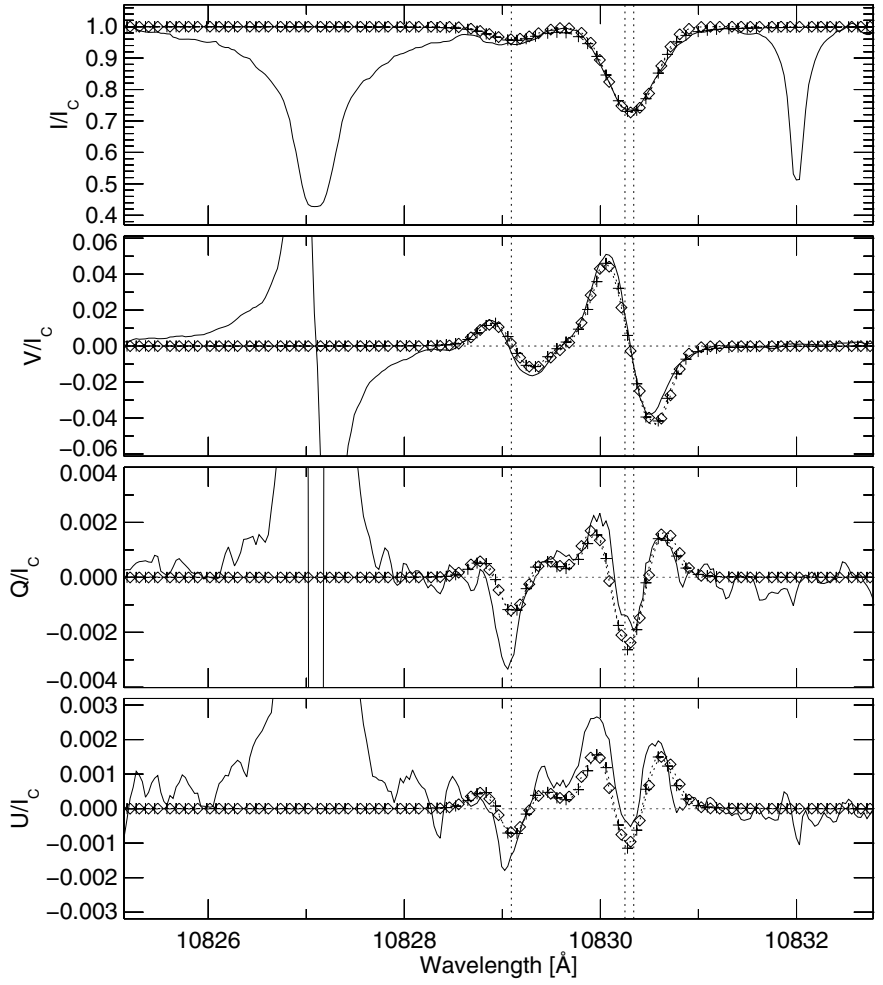

Fig. 4. Effect of a fixed or free damping constant on the profile shape. The reference direction for $Q$ is along the $+y$-axis of the maps in Fig. 1. The observed profile (solid line, pixel $x=26, y=11$ ) is from the center of the small pore, where He I absorption and the magnetic field are strong. The profile with the damping constant as a free parameter (diamonds) is almost indistinguishable from the one where the damping is fixed at a small value (plus signs). The relevant atmospheric parameters retrieved by the fits are very similar (see Table 2).

The telluric blend at $1083.2 \mathrm{~nm}$ was excluded by not using the $I$-profile for $\lambda \geq 1083.135 \mathrm{~nm}$. For the PIKAIA algorithm we define the fitness function as $f=1 / \delta$.

The PIKAIA genetic algorithm allows to specify a range for every parameter to be fitted. This guarantees that the result of the inversion stays within the regime of physically useful solutions. The parameter ranges are given in Table 2 (second column).

To improve the robustness of the fit routine we applied a "multi-iteration" technique: in a first step we run the inversion with high weights on the Stokes $Q$ and $U$ signal to reliably determine the azimuthal direction. After this inversion we run another inversion with equal weight to all four Stokes parameters, where the azimuthal angle is only allowed to vary $\pm 10^{\circ}$ around the result from the first iteration. This technique turned out to be more robust in finding the global minimum than using only one inversion with double the number of iteration steps.

In order to reduce the number of free parameters we investigated the effect of line broadening due to collisional and radiative damping on the He I lines. Both effects influence the wings of a line more than the core, which is dominated by Doppler broadening. For weak lines like our He I multiplet it is the Doppler core which dominates the shape of the spectral line. Since the collisional and radiative damping are very weak we assume the damping constant $a$ to be almost zero. We therefore
Table 2. Atmospheric parameters for profile x $26-\mathrm{y} 11$.

\begin{tabular}{lccc}
\hline \hline Parameter & $\begin{array}{c}\text { fit range } \\
\text { min-max }\end{array}$ & $\begin{array}{c}\text { value } \\
\text { free } a\end{array}$ & $\begin{array}{c}\text { value } \\
a=10^{-4}\end{array}$ \\
\hline$B$ [Gauss $]$ & $0-2000$ & 1119.5 & 1072.3 \\
$\gamma\left[^{\circ}\right]$ & $0-180$ & 18.3 & 16.9 \\
$\chi\left[^{\circ}\right]$ & $-90-90$ & 36.0 & 37.4 \\
$v_{\text {LOS }}[\mathrm{m} / \mathrm{s}]$ & $-10000-40000$ & 358.3 & 396.4 \\
$a$ & $0-0.5$ & 0.392 & 0.0001 \\
$\Delta \lambda_{\mathrm{D}}[\AA]$ & $0-0.7$ & 0.198 & 0.263 \\
$\eta_{0}$ & $0-10$ & 5.330 & 3.52 \\
$\mu S_{1}($ fixed $)$ & $0-5$ & 1.000 & 1.000 \\
fitness & - & 14.71 & 10.65 \\
\hline
\end{tabular}

fix this parameter and set it to $10^{-4}$, which reduces the number of free parameters per atmospheric component to 7. Figure 4 demonstrates the negligible effect of the damping constant on the resulting fits and Table 2 shows that the parameters relevant for our analysis are also very similar. The observed profile in Fig. 4 was taken from the center of the small pore, where the absorption signature is strongest and damping effects could play a role, representing a "worst case scenario" for neglecting the damping constant. The fits obtained with a fixed damping of $10^{-4}$ are slightly worse than the ones where $a$ was treated as a free parameter. However, the relevant atmospheric parameters $B, \gamma, \chi$ and $v_{\text {LOS }}$ returned by both fits are almost unaffected by this choice (see Table 2 ). In the region between the two pores exhibiting only weak Stokes profiles the difference is even smaller. Since the stability of the inversion results increases when eliminating $a$ as a free parameter, in our further analysis we use a fixed damping constant. Note that the thermodynamic parameters $a, \Delta \lambda_{\mathrm{D}}$ and $\eta_{0}$, which are not of further interest to us, are affected strongly by this decision. When leaving $a$ free, the returned values of these thermodynamic parameters fluctuate strongly from one profile to another. They are obviously not completely independent of each other and fixing $a$ leads to more stable results for $\Delta \lambda_{\mathrm{D}}$ and $\eta_{0}$ as well.

The simple implementation of the Hanle effect is demonstrated in Fig. 5. For the few profiles where we can observe both - a broad signal in $U$ due to the scattering polarization overlaid with a typical Zeeman signal in $Q, U$, and $V$ - we obtain consistent information for the magnetic field azimuthal direction. In the example presented in Fig. 5 (pixel $x=58, y=28$ in Fig. 1) the azimuthal angle when neglecting the Hanle effect is almost unconstrained, whereas the combined Zeeman-Hanle diagnostics gives a very stable result of $\chi=-49^{\circ}$. This is very close to the value deduced from nearby Stokes profiles that are dominated either by the Zeeman effect or the Hanle effect. This $\chi$ value is also consistent with a field directed along the fibrillike structures in the line center intensity map in Fig. 1.

Another simplification concerned the gradient of the source function $S_{1}$. The inversion of a large number of measured profiles showed, that the line center absorption coefficient $\left(\eta_{0}\right)$ strongly competed with $S_{1}$, leading to an ambiguity in the 


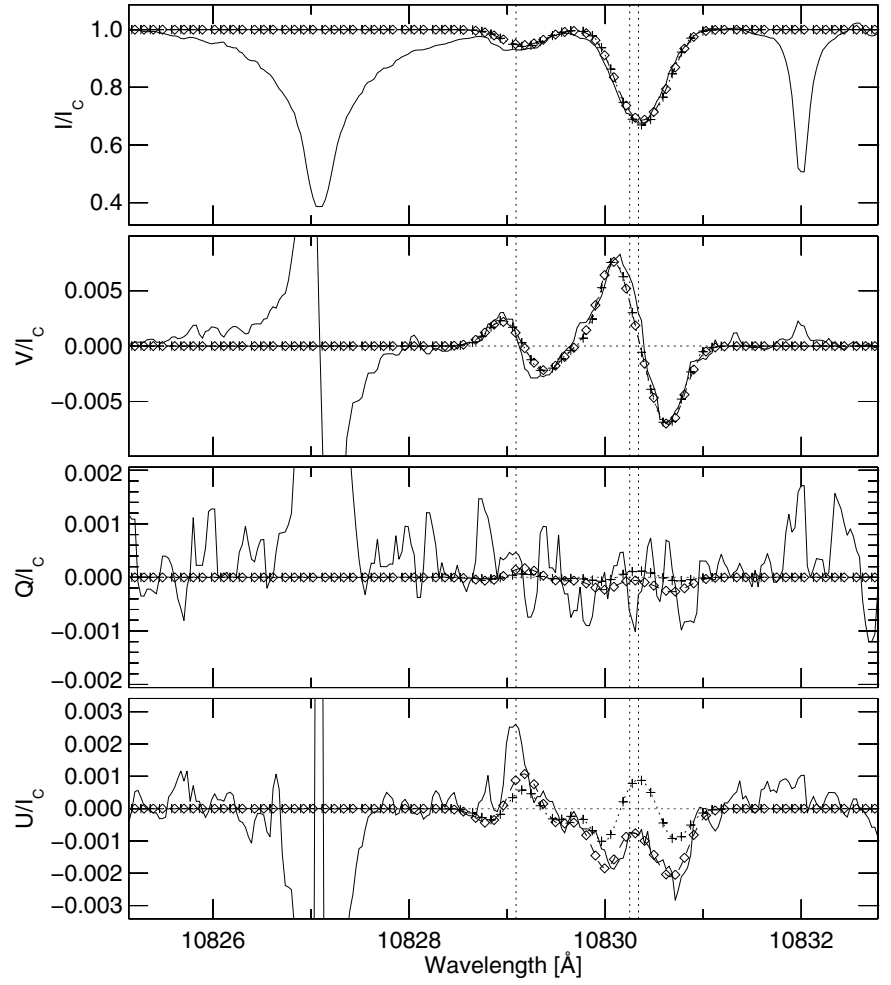

Fig. 5. Combined Hanle and Zeeman diagnostics. The synthetic $Q$ and $U$ profiles obtained using the combination of Zeeman-diagnostics and the special case of the Hanle effect valid close to disk center (diamonds) agree very well with the observed profile at $x=58, y=28$ (solid line). For the case where the Hanle effect is not included (plus signs), the fit to the observed $U$ profile is unsatisfactory. The reference direction for Stokes $Q$ is along the $+y$-axis of the maps in Fig. 8 .

determination of these parameters. Apparently there is not enough information in the data to independently determine both parameters. Both parameters increase the strength of the absorption profiles with only minor differences in the profile shape. For the further analysis we therefore set $\mu S_{1}$ to unity. Since we do not derive any physical parameter from $\eta_{0}$ and the source function gradient we regard this simplification as justified.

\section{Convergence and noise analysis}

\subsection{Synthetic profiles with artificial random noise}

The stability of our inversion code when faced with noisy data was tested by inverting synthesized profiles with added artificial random noise. We concentrated on the reliability of the determination of the magnetic field strength and direction. Figure 6 summarizes the results of this test. The parameter values used to synthesize the profiles which then act as artificial data (Table 3) are compared with the results of the inversion. Note that the inclination angle $\gamma$ was set to $80^{\circ}$, which represents a worst case scenario for the determination of the magnetic field strength, since the $V$-signal becomes very weak. Every polar plot, summarizing the scatter of the results from 200 inversions of the synthesized profiles with added artificial random noise, is divided into two regions: the upper

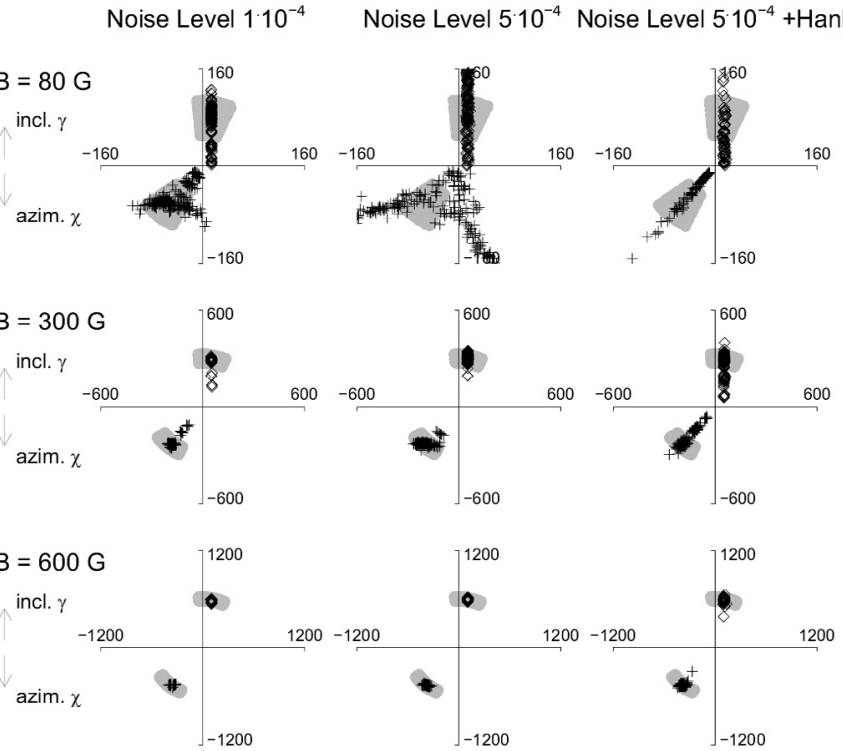

Fig. 6. Convergence and noise analysis of synthesized spectra for the parameters magnetic field strength $(B)$ and direction $(\chi$ and $\gamma)$. Every polar plot is divided in two regions: the upper half shows as radial coordinate the magnetic field strength $B$ (in Gauss) with the inclination $\gamma$ as polar angle (diamonds). The lower half is similar but with the azimuthal angle $\chi$ as polar angle (plus signs). The gray shaded areas indicate the magnetic field vector used for the synthesis with a deviation of $\pm 15^{\circ}$ for the angles and $\pm 30 \mathrm{G}$ in strength around the initial values in Table 3. The three rows stand for synthetic profiles calculated for different magnetic field strengths of $80 \mathrm{G}, 300 \mathrm{G}$ and $600 \mathrm{G}$. Random noise with an amplitude of $10^{-4} I_{\mathrm{c}}$ (left column) and $5 \times 10^{-4} I_{\mathrm{c}}$ (middle and right column) was added to the synthesized profiles. The right column displays the results using, in addition to the Zeeman effect, the simple implementation of the Hanle effect leading to a very reliable determination of the magnetic field direction.

half shows the magnetic field strength $B$ (in Gauss) as radial coordinate, with the inclination $\gamma$ as polar angle (diamonds). The lower half is similar but with the azimuthal angle $\chi$ (plus signs) as the polar angle. The three rows of Fig. 6 represent the results for different initial magnetic field strength (from top to bottom: $80 \mathrm{G}, 300 \mathrm{G}$ and $600 \mathrm{G}$ ). The left and the center columns show the inversion results for low $\left(10^{-4} I_{\mathrm{c}}\right)$ and typical $\left(5 \times 10^{-4} I_{\mathrm{c}}\right)$ noise levels added to a synthesized profile where the amplitude due to scattering polarization, $H_{0}$, was set to zero. Column three summarizes the convergence for a noise level of $5 \times 10^{-4} I_{\mathrm{c}}$. Here an artificial scattering-polarization signal with an amplitude of 0.002 was added to the Stokes $Q$ and $U$ profiles according to Eq. (24). The initial parameters for the synthesis are summarized in Table 3 . Note that only a single atmospheric component is assumed to be present.

The scatter in the inversion results originates from the artificial random noise which is different for every run, and in the genetic algorithm used for minimization: after a finite number of iteration steps the genetic algorithm does not always reach the global maximum. The variation of the convergence for 200 test runs gives an idea of the reliability of the value returned by the inversion. We do not show the values for the line-of-sight velocity $v_{\mathrm{LOS}}$ and for the Doppler broadening $\Delta \lambda_{\mathrm{D}}$, since they are reproduced with an accuracy of $\$ 2 \%$ of the total fit range (see 
Table 3. Parameters for synthesized profile used for convergence and noise analysis.

\begin{tabular}{lc|lc}
\hline \hline Parameter & value & Parameter & value \\
\hline$B$ [Gauss $]$ & $80 / 300 / 600$ & $v_{\text {LOS }}[\mathrm{m} / \mathrm{s}]$ & 5000 \\
$\gamma\left[^{\circ}\right]$ & 80 & $\chi\left[^{\circ}\right]$ & -40 \\
$\eta_{0}$ & 1.0 & $\Delta \lambda_{\mathrm{D}}[\AA]$ & 0.250 \\
$a$ & 0.0001 & $\mu S_{1}$ & 1.0 \\
\hline
\end{tabular}

Table 2). For simplicity, we assumed the gradient of the source function to be 1 (this assumption turned out to be consistent with all inverted pixels of our observation) and the damping constant $a=10^{-4}$.

Figure 6 clearly shows that the determination of the magnetic field strength and direction is very accurate for the Zeeman-dominated regime ( $B=600 \mathrm{G}$, 3rd row). Here the magnetic field direction returned by the inversion lies within $\pm 5^{\circ}$, the magnetic field strength within $\$ 20 \%$ of the initial value. Decreasing the magnetic field strength underlying the artificial data increases the uncertainty in the determination of the magnetic field direction and strength. Especially in the weak field case (1st row) the rms error in the magnetic field strength increases up to $\pm 100 \mathrm{G}$ because the polarization signal approaches the noise level (signal to noise ratio $\approx 2$ ). The typical noise level case $\left(5 \times 10^{-4} I_{\mathrm{c}}, 2\right.$ nd column $)$ does not allow for a reliable determination of the azimuthal angle. The presence of a Hanle effect signal (3rd column) significantly improves the determination of the azimuthal angle and leads to an accuracy of $\pm 5^{\circ}$. The inclination angle $\gamma$ (top half of the polar plots) is retrieved reasonably well in all cases. We expect that a more realistic implementation of the Hanle effect by calculating the full polarization diagrams would also lead to a significant improvement of the determination of the magnetic field strength in the regime of weak fields below $200 \mathrm{G}$, at locations where the Hanle signal is detectable (Trujillo Bueno et al. 2002).

\subsection{PIKAIA}

Genetic algorithms have several advantages compared to standard minimization techniques. They do not require the calculation of derivatives of the model parameters in the fit function and are very robust in finding the global minimum of a multidimensional hypersurface independent of the initial parameter values. We decided to use the code PIKAIA (Charbonneau 1995), since it is a very well written and easy to use public domain code available in Fortran and IDL. To demonstrate its superior robustness we compared the results of the genetic algorithm with the ones obtained with UOBYQA (Unconstrained Optimization BY Quadratic Approximation, Powell 2002), a code which estimates the first and second derivatives of the parameters in the fitness function using quadratic approximations. This code was used for comparison because it provides an interface to the function to be optimized which is very similar to PIKAIA.

The low signal to noise ratio, especially in Stokes $U$ and $Q$, sets high demands on the robustness of the optimization routine. The parameter mainly affected by these two profiles is the azimuthal angle of the magnetic field, $\chi$. Maps of this parameter therefore are the best indicator for the robustness of the used optimization method. Figure 7 presents these maps using PIKAIA (top) and UOBYQA (bottom panel). The superior robustness of the PIKAIA-based inversion manifests itself in the much smoother transition of the magnetic field azimuth between the two pores of opposite magnetic polarity. We preferred the robustness of the genetic algorithm to the speed increase of a factor $\gtrsim 10$ when using the quadratic approximation technique.

\section{Application: Emerging flux region}

\subsection{One-component model atmosphere}

For most of the pixels in Fig. 1 an inversion based on an atmosphere with one atmospheric component, a magnetic component, gives good results. A combination of a magnetic and a field-free component gives very similar results, although with much greater uncertainty in the field strength, which cannot be well distinguished from the filling factor in this case. This is because outside the pores the Zeeman splitting is so small that the He I lines are very close to the weak-field limit (Landi Degl'Innocenti \& Landi Degl'Innocenti 1973). Obviously there is no great advantage in adding a field-free component to the inversions in this case. A single magnetic component is expected to be sufficient for the upper chromosphere, where the magnetic field fills all available space (e.g. Jones 1985; Solanki \& Steiner 1990). We therefore refrain from introducing a further field-free component. The model atmosphere has 7 free parameters: magnetic field strength and direction $(B, \gamma, \chi)$, line-of-sight velocity $\left(v_{\mathrm{LOS}}\right)$, line center relative absorption coefficient $\left(\eta_{0}\right)$, Doppler broadening $\left(\Delta \lambda_{\mathrm{D}}\right)$ and the amplitude of the scattering polarization signal in linear polarization profiles due to the Hanle effect $\left(H_{0}\right.$, see Sect. 3.2). The free parameters were allowed to vary between the minimum and maximum range shown in Table 2.

The maps for the free parameters of the inversion are displayed in Fig. 8. The top panel clearly identifies two pores through the magnetic field strength which exceeds $1 \mathrm{kG}$ (and their correspondence in darkening of the continuum). The second and third panel show the azimuthal direction and the inclination of the magnetic field. Note that in regions where the magnetic field is sufficiently inclined $\chi$ is returned quite stably, with little variation from pixel-to-pixel. For nearly longitudinal fields $\chi$ is less stable, but is almost irrelevant, since even large errors in $\chi$ at such locations imply only small errors in the direction of the magnetic field vector. The line-of-sight velocity map is displayed in panel 4 with red (blue) shaded regions marking downflows (upflows). It shows a considerable asymmetry between up and downflows. A region of slowly upward moving gas in the center is flanked by downflowing material reaching supersonic velocities up to $25 \mathrm{~km} \mathrm{~s}^{-1}$. The Doppler broadening in panel 5 is indicative of a low turbulent velocity in the slowly upward moving gas if one assumes that the temperature of formation is roughly the same everywhere. Note, 

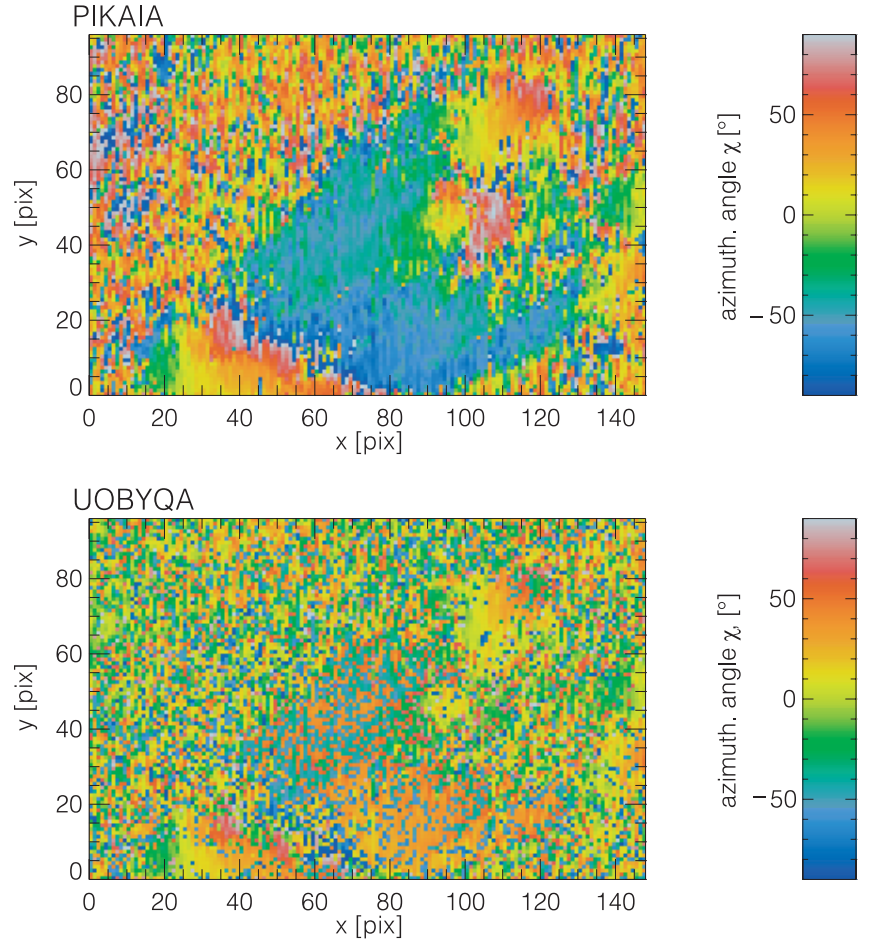

Fig. 7. Inversion results for the azimuthal angle $(\chi$, reference direction $+y$-axis) using the genetic algorithm PIKAIA (top panel) and UOBYQA (bottom panel). Large pixel to pixel variation using UOBYQA indicate that the optimization routine got stuck in a local minimum of the parameter space.

however, that the $\Delta \lambda_{\mathrm{D}}$ value returned by the code is affected significantly by the choice of damping parameter $a$.

All these facts are consistent with the picture of freshly emerging magnetic flux tubes which rise in magnetic loops while conserving the relatively cool photospheric and slightly subphotospheric temperature. The $\eta_{0}$ value plotted in panel 6 shows fibril-like structures connecting the two regions of opposite magnetic polarity. These fibrils correlate very nicely with the direction of the magnetic field and with the line-center linestrength (top panel of Fig. 1). The amplitude of scattering polarization in panel 7 shows where the magnetic azimuth is most stably retrieved by the Hanle diagnostics. Here the magnetic field inclination is nearly horizontal, as required for the simple implementation of the Hanle effect. The magnetic field direction of this region smoothly connects to the regions dominated by the Zeeman effect. This result confirms the applicability of the used approximation for the Hanle effect. The last panel shows the fitness $f=1 / \delta$ of the PIKAIA-fits (see Eq. (25)). Higher values indicate a better fit than lower values. The poor fits in the pore in the lower left corner are mainly due to the presence of two velocity components within one pixel (see Sect. 6.2). Table 2 shows that the fitness in this region depends significantly on whether magnetooptical effects are taken into account or not.
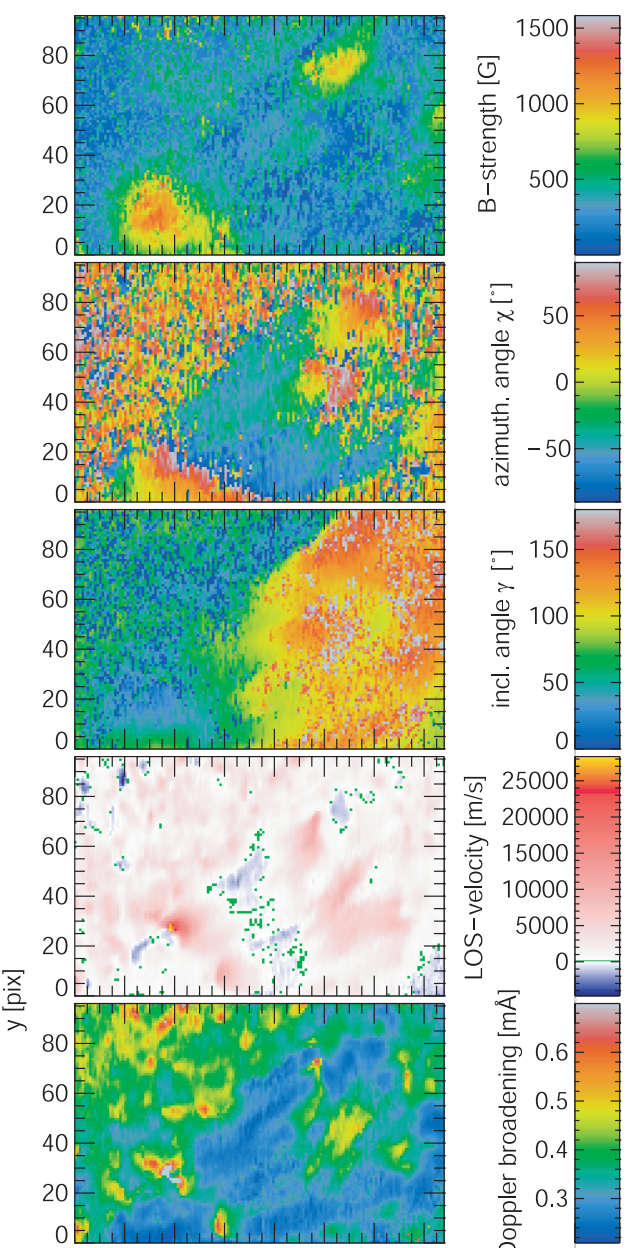

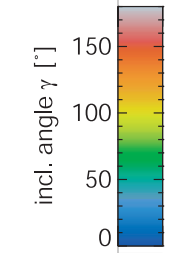
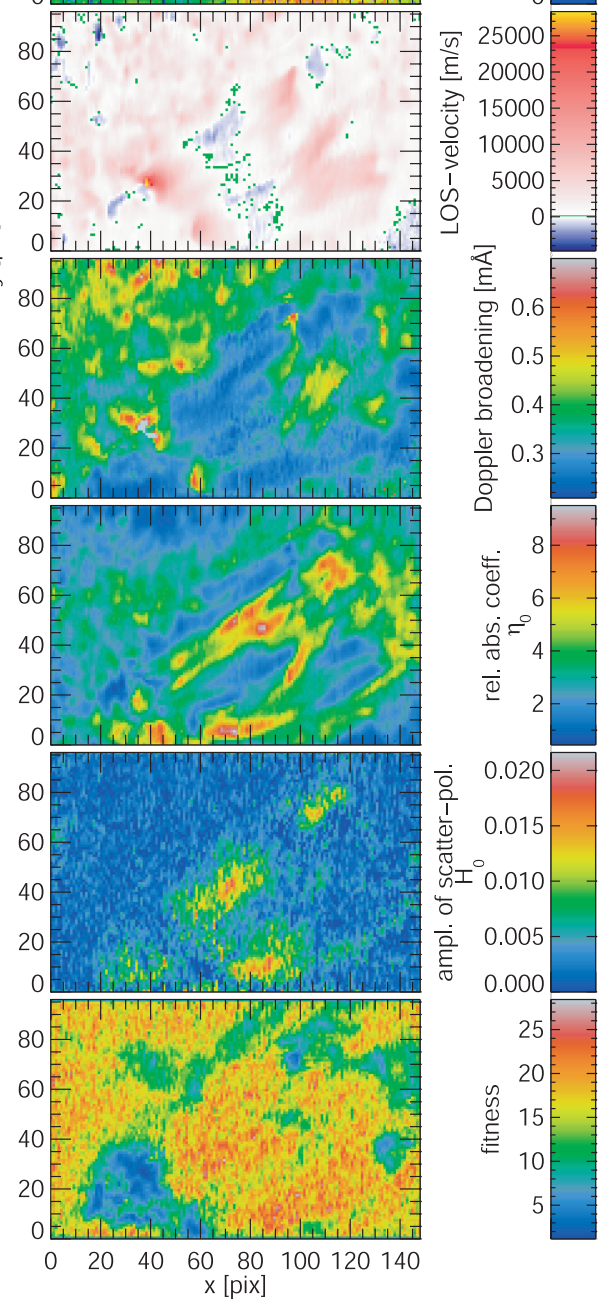

Fig. 8. Atmospheric parameters returned by a 1-component inversion. The reference direction for the azimuth (2nd panel) is the $+y$-axis.

\subsection{Two-component atmosphere}

In order to obtain optimum fits throughout the scan we repeated the inversion employing two magnetic components (allowing each to harbor different line-of-sight flows). Since the 


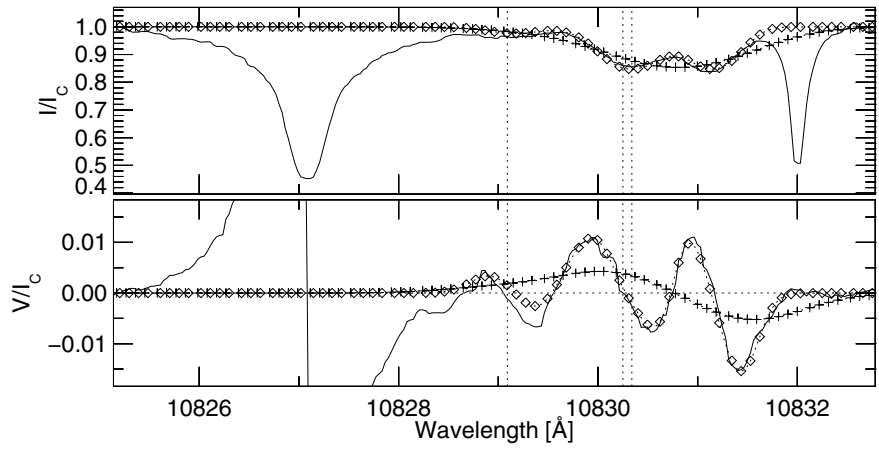

Fig. 9. Comparison of 1-component (plus signs) and 2-component (diamonds) fits to the $I$ and $V$ profiles at pixel $x=40, y=25$ (solid line). Clearly the 1-component fit is not able to reproduce the data reasonably, whereas the synthetic profiles resulting from a 2-component fit very nicely match the observations.

Table 4. Parameters returned by the 1-component and 2-component fit to pixel $x=40, y=25$.

\begin{tabular}{lccc}
\hline \hline Parameter & 1-component & \multicolumn{2}{c}{ 2-component } \\
Parameter & & comp. 1 & comp. 2 \\
\hline$B$ [Gauss] & 730.28 & 714.36 & 774.64 \\
$\chi\left[^{\circ}\right]$ & 63.03 & 60.57 & 60.57 \\
$\gamma\left[^{\circ}\right]$ & 32.89 & 41.17 & 41.17 \\
$v_{\mathrm{LOS}}[\mathrm{m} / \mathrm{s}]$ & 14933.49 & 1799.36 & 23869.20 \\
$\Delta \lambda_{\mathrm{D}}[\AA]$ & 0.91 & 0.33 & 0.30 \\
$\eta_{0}$ & 1.24 & 3.86 & 3.86 \\
filling factor $\alpha$ & 1.00 & 0.48 & 0.52 \\
$a$ (fixed) & 0.0001 & 0.0001 & 0.0001 \\
fitness & 3.2 & \multicolumn{2}{c}{15.7} \\
\hline
\end{tabular}

Stokes $Q$ and $U$ profiles are often too weak and noisy to allow the transverse magnetic field of the two atmospheric components to be separated we reduce the number of free parameters for the 2-atmosphere inversion by forcing the magnetic field direction to be equal for both atmospheres. As an example, both 1-component and 2-component fits to a complex $I$ and $V$ spectrum of the He lines are displayed in Fig. 9. The spectrum was recorded close to one of the pores (pixel $x=40$, $y=25$ in Fig. 1). Figure 9 and Table 4 demonstrate that in certain regions, specifically right at the edges of the pore, the Helium lines clearly sample two magnetic atmospheres with different velocities which coexist within a single pixel of the measurements, a non Doppler-shifted (or only weakly shifted) atmosphere and a significantly shifted atmosphere. The photospheric Si I line inverted with SPINOR exhibits no such signature (not shown). The fits to the He I multiplet are satisfactory (and the fitness greatly improved - see Table 4) if two magnetic components are employed.

The parameters returned by the 1- and 2-component fits are summarized in Table 4. The 1-component fit tries to account for the second component by increasing the Doppler broadening to an unrealistically high value. Obviously the resulting information on magnetic field direction and strength does not reflect the real conditions. The 2-component fit clearly identifies a slow component $\left(1.8 \mathrm{~km} \mathrm{~s}^{-1}\right.$ downflow) and a fast component (23.8 $\mathrm{km} \mathrm{s}^{-1}$ downflow). The magnetic field strength in both components is almost identical.

The fact that neighboring profiles give very similar results from 2-component inversions, with the returned parameter varying smoothly from pixel to pixel, reinforces our belief in the robustness of the results. A more complete and thorough analysis of the data (including time series) exhibiting strong downflows and requiring 2-component fits will be the subject of a separate publication (Lagg et al., in preparation).

\section{Summary and outlook}

We have presented an extension of techniques previously used to study the He I $1083 \mathrm{~nm}$ multiplet such that spectral line saturation (using a simple Milne-Eddington approach, extended to allow for blending lines) and magnetooptical effects are taken into account. By combining the Unno-Rachkowsky solutions of the radiative transfer equations for polarized light with a simple representation of the Hanle effect (valid for horizontal fields near solar disk center) and with a minimization scheme based on genetic algorithms it is possible to determine the full magnetic vector (although the $180^{\circ}$ uncertainty inherent in all Zeeman effect diagnostics still remains) together with the lineof-sight velocity and auxiliary quantities such as the Doppler width (the last quantity with lower reliability). In some locations the Hanle effect dominates the net linear polarization signal and its introduction into the inversion scheme helps to make the results more robust.

Tests of the inversion code on artificial and real data have been very encouraging. In particular, the use of a genetic algorithm significantly reduces the dependence of the final results on the initial guess, since it searches for the absolute minimum of a function describing the error in the fit. This removes one of the main shortcomings of Stokes profile inversions, namely that the minimization routine ends up finding different minima (and hence different sets of "best fit" parameters depending on the initial guess). A genetic algorithm would greatly strengthen the reliability of Milne-Eddington inversions of other spectral lines as well, such as the lines regularly observed by the Advanced Stokes Polarimeter (ASP, Elmore et al. 1992). Its use for inversions employing numerical solutions of the transfer equation will certainly increase with the available computing power.

The inversion code we have written also allows for the possible presence of multiple atmospheric components (with the freedom to couple individual parameters between different components if the information content of the data is not sufficiently high). Two component fits of profiles with a non-standard shape give reasonable and stable results. The most obvious next improvement in the analysis procedure is full treatment of the Hanle and Zeeman effects as done by Trujillo Bueno et al. (2002) for solar coronal filaments. Nonetheless, the technique is now ripe to be employed to analyze solar data in order to profit from the unique diagnostic capabilities of the He I multiplet at $1083 \mathrm{~nm}$. A first such analysis has been reported by Solanki et al. (2003), others are currently in the process of being carried out. 
Acknowledgements. The authors would like to thank Manolo Collados for his help during the observing run. We also thank Javier Trujillo Bueno for helpful discussions regarding the Hanle effect and Paul Charbonneau for providing the reliable and easy to use genetic algorithm PIKAIA.

\section{References}

Auer, L. H., House, L. L., \& Heasley, J. N. 1977, Sol. Phys., 55, 47 Avrett, E. H., Fontenla, J. M., \& Loeser, R. 1994, in Infrared Solar Physics, ed. D. M. Rabin (Dordrecht: Kluwer Academic Publishers), IAU Symp., 154, 35

Bommier, V., Sahal-Brechot, S., \& Landi Degl'Innocenti, E. 1991, A\&A, 244, 383

Charbonneau, P. 1995, A\&AS, 101, 309

http://www.hao.ucar.edu/public/research/si/pikaia/ pikaia.html

Collados, M. 2001, in Advanced Solar Polarimetry - Theory, Observation, and Instrumentation, ASP Conf. Ser., 236, 255

Collados, M., Trujillo Bueno, J., \& Asensio Ramos, A. 2003, in Solar Polarization 3, ed. J. Trujillo Bueno, \& J. Sanchez Almeida, ASP Conf. Ser., 307, 468

Condon, E. U., \& Shortley, G. H. 1963, The theory of atomic spectra (Cambridge: University Press)

del Toro Iniesta, J. 2003, Introduction to Spectropolarimetry No. ISBN 0521818273 (Cambridge University Press)

Elmore, D. F., Lites, B. W., Tomczyk, S., et al. 1992, in Polarization analysis and measurement; Proc. Meeting, San Diego, CA, July 19-21, 1992 (A93-33401 12-35), ed. D. H. Goldstein, \& R. A. Chipman, SPIE, 1746, 22-33

Falconer, D. A., Moore, R. L., Porter, J. G., Gary, G. A., \& Shimizu, T. 1997, ApJ, 482, 519

Faurobert-Scholl, M. 1992, A\&A, 258, 521

Frutiger, C. 2000, Ph.D. Thesis, ETH Zürich, Switzerland, Diss ETH No. 13896

Frutiger, C., Solanki, S. K., Fligge, M., \& Bruls, J. H. M. J. 2000, A\&A, 358, 1109

Giovanelli, R. G., \& Hall, D. 1977, Sol. Phys., 52, 211

Happer, W. 1972, Rev. Mod. Phys., 44, 169

Harvey, J., \& Hall, D. 1971, in Solar Magnetic Fields, ed. R. Howard (Dordrecht: Reidel), IAU Symp., 43, 279

Jefferies, J., Lites, B. W., \& Skumanich, A. 1989, ApJ, 343, 920

Jones, H. P. 1985, in Chromospheric Diagnostics and Modelling, ed. B. W. Lites, National Solar Obs., Sacramento Peak, New Mexico, 175

Jones, H. P., Duvall, T. L., Harvey, J. W., et al. 1992, Sol. Phys., 139, 211

Landi Degl'Innocenti, E. 1976, A\&AS, 25, 379

Landi Degl'Innocenti, E. 1982, Sol. Phys., 79, 291

Landi Degl'Innocenti, E., \& Landi Degl'Innocenti, M. 1973, Sol. Phys., 31, 299
Lin, H., Penn, M. J., \& Kuhn, J. R. 1998, ApJ, 493, 978

Lites, B. W., Elmore, D. F., Seagraves, P., \& Skumanich, A. P. 1993, ApJ, 418, 928

López Ariste, A., \& Casini, R. 2002, ApJ, 575, 529

Martínez Pillet, V., Collados, M., Sánchez Almeida, J., et al. 1999, in High Resolution Solar Physics: Theory, Observations, and Techniques (Astronomical Society of the Pacific), ASP Conf. Ser., 183,264

Nagendra, K. N., Frisch, H., \& Faurobert-Scholl, M. 1998, A\&A, 332, 610

Parker, E. N. 1983a, ApJ, 264, 635

Parker, E. N. 1983b, ApJ, 264, 642

Parker, E. N. 1988, ApJ, 330, 474

Penn, M. J., \& Kuhn, J. R. 1995, ApJ, 441, L51

Powell, M. J. D. 2002, Math. Programming, 92, 555

Rachkowsky, D. N. 1962, Izv. Krym. Astrofiz. Obs., 27, 148

Rachkowsky, D. N. 1967, Izv. Krym. Astrofiz. Obs., 37, 56

Régnier, S., Amari, T., \& Kersalé, E. 2002, A\&A, 392, 1119

Rüedi, I., Keller, C. U., \& Solanki, S. K. 1996, Sol. Phys., 164, 265

Rüedi, I., Solanki, S. K., \& Livingston, W. C. 1995, A\&A, 293, 252

Scherrer, P. H., Bogart, R. S., Bush, R. I., et al. 1995, Sol. Phys., 162, 129

Schrijver, C. J., Title, A. M., Berger, T. E., et al. 1999, Sol. Phys., 187, 261

Seehafer, N., \& Staude, J. 1979, Astron. Nachr., 300, 151

Socas-Navarro, H., Trujillo Bueno, J., \& Ruiz Cobo, B. 2000a, Science, 288, 1396

Socas-Navarro, H., Trujillo Bueno, J., \& Ruiz Cobo, B. 2000b, ApJ, 530, 977

Solanki, S. K., Keller, C., \& Stenflo, J. O. 1987, A\&A, 188, 183

Solanki, S. K., Lagg, A., Woch, J., Krupp, N., \& Collados, M. 2003, Nature, 425

Solanki, S. K., Rüedi, I. K., \& Livingston, W. 1992, A\&A, 263, 312

Solanki, S. K., \& Steiner, O. 1990, A\&A, 234, 519

Stenflo, J. O. 1994, Solar magnetic fields: polarized radiation diagnostics (Astrophysics and Space Science Library, Dordrecht: Kluwer Academic Publishers)

Stenflo, J. O., Solanki, S., Harvey, J. W., \& Brault, J. W. 1984, A\&A, 131,333

Trujillo Bueno, J. 2001, in Advanced Solar Polarimetry - Theory, Observation, and Instrumentation, ASP Conf. Ser., 236, 161

Trujillo Bueno, J. 2003, in Solar Polarization 3, ed. J. Trujillo Bueno, \& J. Sanchez Almeida, ASP Conf. Ser., 307, 407

Trujillo Bueno, J., Landi Degl'Innocenti, E., Collados, M., Merenda, L., \& Manso Sainz, R. 2002, Nature, 415, 403

Unno, W. 1956, PASJ, 8, 108

White, S. M. 2002, Astron. Nachr., 323, 265

White, S. M., \& Kundu, M. R. 1997, Sol. Phys., 174, 31

Yan, Y., \& Sakurai, T. 1997, Sol. Phys., 174, 65 